\title{
Theory of Chronoamperometric Curves for a Short Time at Microband Electrodes
}

\author{
Koichi AOKI*, Koichi TOKLDA and Hiroaki MATSLDA \\ Received August 15, 1986 ; Accepted September 4, 1986
}

\begin{abstract}
An expression for chronoamperometric curves at a microband electrode was analytically derived by solving the time-dependent two-dimensional diffusion equation. The Wiener-Hopf method, which is an application of the techniques of the Fourier transformation, was employed for solving the initial and boundary value problem. The expression thus obtained was an expansion valid for a short electrolysis time. The leading term of the expansion was cottrellian while the second term was independent of the time. The chronoamperometric curve calculated from the analytical expression was in good agreement with the curve evaluated from the digital simulation.
\end{abstract}

\section{INTRODUCTION}

An interesting feature of ultramicroelectrodes is enhancement of the current density due to the edge effect or two- or three-dimensional diffusion ${ }^{1)}$. Electrodes at which the edge effect is most appreciable are a microshperical electrode and a microdisk electrode. The steady state current can be observed at these electrodes even in a quiescent solution because of an enhancement of the mass transport of electroactive species due to three-dimensional diffusion. Above all, the microdisk electrode can readily be fabricated to have the ideal geometry. Thus it has been extensively applied to in vivo measurements as well as studies on electrode kinetics. Theoretical works for this electrode have been devoted to chronoamperometry", linear sweep voltammetry ${ }^{3)}$, chronopotentiometry ${ }^{4)}$, and square wave voltammetry $y^{5}$, . On the other hand, electrodes with a slightly weak contribution of the edge effect are a microcylinder electrode and a microband electrode, which are characterized by mass transport due to two-dimensional diffusion. These electrodes exhibit no steady state current even at a long time but have the quasisteady state current with a logarithmic timedependence ${ }^{7)}$. When the microcylinder or the microband electrode is so long that a surface area of the electrode is similar to that of an electrode with a conventional size, a

\footnotetext{
Department of Electronic Chemistry, Graduate School at Nagatsuta, Tokyo Institute of Technology, Nagatsuta, Midoriku, Yokohama 227

Key words: Microelectrodes, Current-time curves
} 
reduction of the total current by diminishing an electrode size can be avoided. Thus, from the analytical viewpoint, a microcylinder and a microband electrodes prevail over microdisk electrodes. Although the mass transport for the microcylinder electrode is two-dimensional diffusion, it can mathematically result in the one-dimensional diffusion problem by use of the cylindrical coordinates. Several theoretical studies have been given to chronoamperometry ${ }^{8)}$, linear sweep voltammetry", hronopotentiometry ${ }^{10)}$ normal and differential pulse voltammetry ${ }^{11)}$, and pulse voltammetry ${ }^{12)}$ for the electrode kinetics and linear sweep voltammetry for the electrode kinetics ${ }^{13)}$.

Recently the microband electrodes have been applied to convenient and sensitive electrochemical detectors which involve the monitoring of transient and steady-state zone distributions of electroactive species in electrophoresis ${ }^{14-19)}$. These detectors are composed of arrays of microband electrodes. The other applications of the microband electrode involve molecular-based microelectronic devices ${ }^{20-26)}$, which have been developed by coating microband electrodes with conductive polymer films.

Though the microband electrode has such fascinating applicabilities, the basic investigation of the microband electrode has been restricted to studies on the limiting behavior of the current decay due to a potential $\operatorname{step}^{27}$. When electrode potential is stepped to the limiting current region, the current decays at first with the cottrellian dependence and then decreases gradually with a logarithmic timedependence ${ }^{7)}$. Oldham ${ }^{28)}$ derived expressions for current density time curves at an extremely thin sheet electrode and applied them to the microband electrode. Since the model is composed of a semi-infinite plane electrode, it cannot directly be applied to the microband electrode. In this paper, we derive expressions for the chronoamperometric curve at the microband electrode when the thickness of the diffusion layer does not exceed the width of the electrode i.e., electrolysis time is relatively short.

\section{DERIVATION}

A scheme of the band electrode is shown in Fig. 1. The band electrode with the width $w$ is located in the domain

$[-w / 2 \leqq x \leqq w / 2$ and $y<0]$ interposed between two insulators. The space for $y>0$ is filled with a solution. It is assumed that the width of the electrode is much smaller than the length (in the direction perpendicular to the $x y-p$ lane). We are concerned with twodimensional diffusion of the electroactive

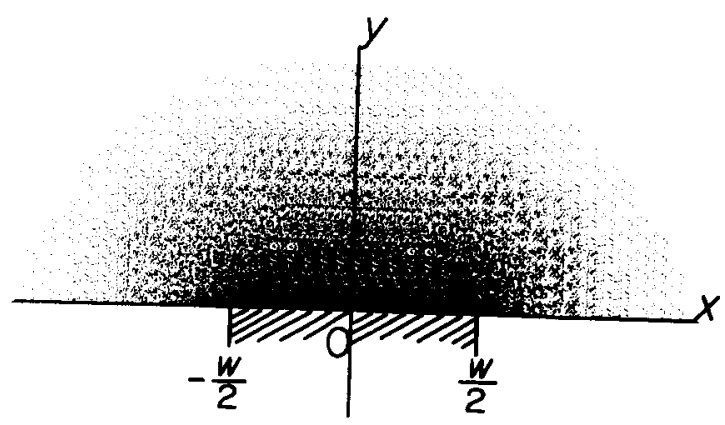

Fig. 1. Coordinates for the microband electrode and concentration profiles obtained by the digital simulation at $\theta=0.1$. 
species without convection or migration when the electrode potential is stepped to the limiting current region. Then the concentration of the species $c$ being a function of $x$, $y$ and the electrolysis time $t$, obeys the following diffusion equation:

$\partial c / \partial t=D\left(\partial^{2} c / \partial x^{2}+\partial^{2} c / \partial y^{2}\right)$

where $D$ is the diffusion coefficient of the electroactive species. The initial and the boundary conditions are given by

$\mathrm{t}=\mathrm{0} \quad: \mathrm{c}=\mathrm{c}^{\circ}$

$t>0, x \rightarrow \pm \infty, y \rightarrow \infty \quad: c \rightarrow c^{*}$

$t>0,-w / 2 \leqq x \leqq w / 2, y=0: c=0$

$t>0,-w / 2 \leqq x \leqq w / 2, \quad y=0: D(\partial c / \partial y)=j / n F$

$t>0, x<-w / 2, x>w / 2, y=0: \partial c / \partial y=0$

where $c^{*}$ denotes the bulk concentration, $j$ the current density, $n$ the number of electrons transferred and $F$ the Faraday constant. Condition (4) denotes the limiting current condition.

Applying the Laplace transformation to eqn. (1) with consideration of eqn. (2) yields $(s / D)\left(\bar{c}-c^{*} / s\right)=\partial^{2} \bar{c} / \partial x^{2}+\partial^{2} \bar{c} / \partial y^{2}$

where s denotes the variable of the Laplace transformation with respect to $t$ and $\bar{c}$ the Laplace transformed concentration. We introduce the Fourier transformation, defined by $F=F(p, y)=(2 \pi)^{-1 / 2} \int_{-\infty}^{\infty}\left(\bar{c}-c^{*} / s\right) e^{i p x} d x$ where $i^{2}=-1$, to eqn. (7) and obtain an ordinary differential equation:

$d^{2} F / d y^{2}=(s / D) F+p^{2} F$ that can be derived from the combination of eqn. (3) and integration by parts

$(2 \pi)^{-1 / 2} \int_{-\infty}^{\infty}\left(\partial^{2}\left(\bar{c}-c^{*} / s\right) / \partial x^{2} 1 e^{j p x} d x=-p^{2} F\right.$

The solution of eqn. (9) is given by

$F(p, y)=A \exp \left[-\left(p^{2}+s / D\right)^{1 / 2} y\right]$

where $A$ is a constant. By differentiating eqn. (11) with respect to $y$, eliminating $A$ from the resulting equation and eqn. (11) and taking $y$ to be 0 , we obtain $\left(p^{2}+s / D\right)^{1 / 2} F(p, 0)+[d F(p, y) / d y]_{y=0}=0$ When we rewrite eqn. (12) as the integral representation through the use of eqn. (8) and insert conditions $(4)-(6)$ into the resulting equation, we obtain

$\int_{-\infty}^{-w / 2}\left((\bar{c})_{y=0}-c^{*} / s\right) e^{i p x} d x$

$-\left(2 c^{*} /(p s) i \sin (p w / 2)+\int_{w / 2}^{\infty}\left((\bar{c})_{y=0}-c^{*} / s\right) \theta^{i p x} d x\right.$

$+\left(D^{2}+s / D\right)^{-1 / 2} \int_{-\pi / 2}^{\pi / 2}(\bar{j} / n F D) e^{i p x} d x=0$

where the second term on the left hand side of ean. (13) has come from the integration of $\left(c^{*} / s\right) e^{i p x}$, corresponding to condition (4). Equation (13) contains two unknowns, $(\bar{j} / n F)$ and $(\bar{c})_{y=0^{\circ}}$ The domains of $x$ in which these two variables are unknown do not overlap each other and cover all region of $x$ for the Fourier integral. such a problem can be solved by the Wiener-Hopf method ${ }^{29)}$, which has been successfully applied to the derivation of the expressions for the diffusion controlled current at stationary micro-disk electrodes ${ }^{2}$ and for the convective diffusion controlled current at microchannel electrodes. ${ }^{30)}$ 
Now we introduce the following five new functions :

$F_{+}(p)=\int_{\pi / 2}^{\infty}\left((\bar{c})_{y=0}-c^{*} / s\right) e^{i p(x-\pi / 2)} d x$

$F_{-}(p)=\int_{-\infty}^{-\infty / 2}(\bar{c})_{y=0}-c^{*} / s l e^{i p(x+\pi / 2)} d x$

$F_{0}(p)=\int_{-\pi / 2}^{\pi / 2}(\bar{j} / n F D) e^{i p x} d x$

$M_{+}(p)=\exp (\pi \dot{i} / 4)\left[p+i(s / D)^{1 / 2}\right]^{-1 / 2}$

$M_{-}(p)=\exp (-\pi i / 4)\left[p-i(s / D)^{1 / 2}\right]^{-1 / 2}$

By use of these functions, ean. (13) can be rewritten as

$e^{i w p / 2} F_{+}(p)+e^{-i m p / 2} F_{-}(p)-\left(2 c^{*} /(s p) / \sin (p w / 2)\right.$

$$
+M_{+}(p) M_{-}(p) F_{0}(p)=0
$$

Dividing the terms in eqn. (19) by $e^{i m p / 2} M_{+}$ yields

$F_{+} / M_{+}+e^{-i w p} F / M_{+}-\left(c^{*} /\left(i s p M_{+}\right)\right)\left(1-e^{-i w p}\right)$

$$
+e^{-i m p / 2} M_{-} F_{0}=0
$$

Discussion is here directed to singularities of all the functions in eqn. (20) on the pplane. $M_{+}(p)$ and $M_{-}(p)$ are singular at $p=-i(s / D)^{1 / 2}$ and $p=i(s / D)^{1 / 2}$ (see eqns. (17) and (18)), respectively, which are branch points. We locate the branch cut lines for $M_{+}(p)$ and $M_{-}(p)$ on the half lines of the imaginary axis, $\operatorname{Im}(D)<-R e\left[(s / D)^{1 / 2}\right]$ and $\operatorname{Im}(p)>\operatorname{Re}\left[(s / D)^{1 / 2}\right]$, respectively. Then $M_{+}(p)$ and $M_{-}(p)$ are holomorphic in the domains, $\operatorname{Im}(p)>-R e\left[(s / D)^{1 / 2} \mid\right.$ and $\operatorname{Im}(p)<R e\left[(s / D)^{1 / 2}\right]$, respectively. For an extremely long electrolysis time, the concentration profile at the microband electrode approaches that at the microcylinder electrode. Then it is expressed by ${ }^{31)}$ $\bar{c} \sim\left(c^{*} / s\right)\left[1-(w /(2 x)]^{1 / 2} \exp \left(-(s / D)^{1 / 2}(x-w / 2) \|\right.\right.$ as $\mathrm{x} \rightarrow \infty$

Substitution of ean. (21) into $F_{+}(p)$ indicates that the integration in $F_{+}(p)$ converges when $\operatorname{Re}\left[i p-(s / D)^{1 / 2}\right]<0$ or $\operatorname{Im}(D)>-\operatorname{Re}\left[(s / D)^{1 / 2}\right]$.

Thus $F_{+}(p)$ is holomorphic in the domain, $\operatorname{Im}(p)>-R e\left[(s / D)^{1 / 2}\right]$. When a similar procedure

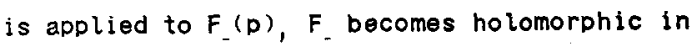
the domain, $\operatorname{Im}(D)<\operatorname{Re}\left[(s / D)^{1 / 2}\right]$. On the other hand, $F_{0}$ is holomorphic in the whole domain of p. It is possible to separate the terms in eqn. (20) into two groups, one being holomorphic for $\left.\operatorname{Im}(p)>-\operatorname{Re} I(s / D)^{1 / 2}\right]$ (on the left hand side of eqn. (22)) and the other being for $\operatorname{Im}(p)<0$ (on the right hand side). Then we have $\mathrm{F}_{+} / \mathrm{M}_{+}+\mathrm{R}_{+}+\mathrm{S}_{+}+\mathrm{T}_{+}$

$=-e^{-i m p / 2} M_{-} F_{0}-R_{-}-S_{-}-T_{-}$

where

$R_{+}+R_{-}=\left(c^{*} /\left(i s p M_{+}\right) e^{-i w p}\right.$

$s_{+}+s_{-}=\theta^{-1 w p_{F}} / M_{+}$

$T_{+}+T_{-}=-c^{*} /($ ispm $)$

Explicit forms of $R_{+}, R_{-1} S_{+}, S_{-1} T_{+}$and

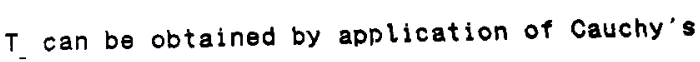
integral theorem to eqns. $(23)-(25)$. Let the integral contours, $C 1$ and $C 2$, be the lines parallel to the real axis, as illustrated in Fig. 2. Then $R_{+1} S_{+}$and $T$, are given by $R_{+}=(1 / 2 \pi i) \int_{C l}\left(c^{*} /\right.$ isz $\left.M_{+}(z)\right) 1 e^{-i n z}(z-p)^{-1} d z(26)$ $s_{+}=(1 / 2 \pi i) \int_{C 1}\left[F_{-}(z) / M_{+}(z)\right) \theta^{-i k z}(z-p)^{-1} d z$ $T_{-}=-(1 / 2 \pi i) \int_{c 2}\left[c^{*} /\left(i s z M_{+}(z) \|(z-p)^{-1} d z\right.\right.$ 
The integrands in eqns. (26) and (27) have a branch point at $z=-i(s / D)^{1 / 2}$, which is the unique singularity on the plane lower than $\mathrm{C} 2$. Therefore the integral path $\mathrm{C} 1$ can be deformed into the contour $\mathrm{C}_{3}$, as shown in Fig. 2. By changing variables $z=r e^{3 \pi i / 2}-i(s / D)^{1 / 2}$ for the contour left to the imaginary axis and $z=r e^{-\pi i / 2}-i(s / D)^{1 / 2}$ for the contour right to the imaginary axis, $R_{+}$is given by $R_{+}=-\left(c^{*} /(\pi s)\right) \int_{0}^{\infty} \sqrt{r} \exp \left[-w r-w(s / D)^{1 / 2}\right]$ $\times\left((r+(s / D))^{1 / 2} H r+(s / D)^{1 / 2}-p i H^{-1} d r\right.$

When the same procedure is applied to

ean. (27), s, becomes

$s_{+}=-(1 / \pi) \int_{0}^{\infty} \sqrt{r} \exp \left[-w r-w(s / D)^{1 / 2}\right]$

$\times p_{+}\left(i r+i(s / D)^{1 / 2}\right)\left(r+(s / D)^{1 / 2}-p i\right)^{-1} d r$

where the following relation has been employed:

$F_{-}(-p)=F_{+}(p)$

When we shift the integral contour $\mathrm{C} 2$ to the upper half plane, retaining the residue at $z=0$,

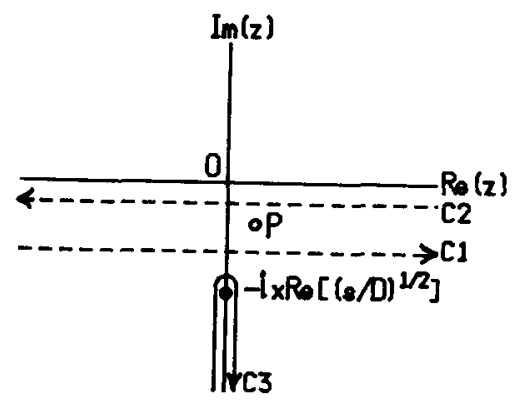

Fig. 2. Integral contours on the z-plane. $c 1$ and $C 2$ are lines parallel to the real axis.

The imaginary part of $\mathrm{C}_{1}$ is between $\operatorname{Im}\left[-i(s / D)^{1 / 2}\right]$ and $\operatorname{Im}(p)$ while that of $\mathrm{C} 2$ is between $\operatorname{Im}(p)$ and 0 . we obtain

$T_{-}=-c^{*} /\{i \operatorname{spM}(0))$

Thus combining eqn. (25) with eqn. (32) yields $T_{+}=\left(c^{*} /(i s p) \mid(s / D)^{1 / 4}-1 / M_{+}(p)\right)$

All the terms on the left hand side of eqn. (22) tend to zero as $p$ approaches infinity between the band domain, $-\operatorname{Re}\left[(s / D)^{1 / 2}\right]<\operatorname{Im}(p)<0$. They are holomorphic in the domain, $\operatorname{Im}(p)>-\operatorname{Re}\left[(s / D)^{1 / 2}\right]$, while the terms on the right hand side of eqn. (22) are holomorphic in the lower half plane. Then application of Liouville's theorem ${ }^{32)}$ to eqn. (22) leads to the fact that the both hand sides of eqn. (22) are zero. Analytical continuation of the left hand side makes the following equation valid for any value of $p$ : $F_{+}(p)=\left\{c^{*} /(i s p) \| 1-(s / D)^{1 / 4} M_{+}(p)\right\}$ $+\left\{M_{+}(p) / \pi\right) \int_{0}^{\infty} \sqrt{r} \exp \left[-w r-w(s / D)^{1 / 2}\right]$

$\times\left\{c^{*} /\left\{s\left(r+(s / D)^{1 / 2}\right)\right\}\right.$

$\left.+F_{+}\left(i r+i(s / D)^{1 / 2}\right)\right]\left(r+(s / D)^{1 / 2}-D i\right)^{-1} d r$

This is an integral equation of the Fredholm type of the second kind. It can be formally solved by the method of successive approximations $^{33)}$. Retaining up to the first term of the Neumann series ${ }^{33)}$ yields

$F_{+}(p)=\left\{c^{*} /(i s p)\right\}\left[1-(s / D)^{1 / 4} M_{+}(p)\right]$

$+\left(c^{*} M_{+}(p) / \pi\right) s^{-3 / 4} D^{-1 / 4} \int_{0}^{\infty} \sqrt{r} \exp \left[-w r-w(s / D)^{1 / 2}\right]$

$\times\left[\left(\left(r+(s / D)^{1 / 2}\right) \|\left(r+(s / D)^{1 / 2}\right)-p i\right)\right.$

$\left.\times\left(r+2(s / D)^{1 / 2}\right]\right]^{-1} d r+0\left(\exp \mid-2(s / D)^{1 / 2} l\right)$

The total current I is expressed by

$I=b \int_{-\varpi / 2}^{w / 2} j d x$ 
where $b$ is the length of the electrode being assumed to be much larger than w. Taking the Laplace transformation of eqn. (36), comparing it with ean. (16) and eliminating $F_{0}(0)$ by use of eqn. (19), we have

$\bar{I} /(n F D b)=F_{0}(0)=c_{*} W(D s)^{-1 / 2}-2(s / D)^{1 / 2} F_{+}(0)(37)$ where we have employed the relation $F_{+}(0)=F_{-}(0)$ (see eqn. (31)). Inserting eqn. (35) into eqn. (37) leads to

$\bar{I} /\left(n F c^{\circ} D b\right)=w(D s)^{-1 / 2}+1 / s$

$-(2 / \pi)(D s)^{-1 / 2} \exp \left[-w(s / D)^{1 / 2}\right]$

$\times \int_{0}^{\infty} \sqrt{r} e^{-m r}\left(\left(r+(s / D)^{1 / 2}\right)\right)^{2}\left(r+2(s / D)^{1 / 2}\right)^{-1 / 2} d r(38)$

We are concerned with the chronoamperometric behavior at a short time, which corresponds to a large value of $|s|$. Expanding the denominator of the integrand in eqn. (38) about $r=0$, retaining only the first term and carrying out the Laplace integration yields $\bar{I} /\left(n F c^{\circ} D b\right)=w(D s)^{-1 / 2}+1 / s$

$$
-(2 \pi)^{-1 / 2} D^{3 / 4} w^{-3 / 2} s^{-7 / 4} \exp 1-w(s / D)^{1 / 2} l+\cdots
$$

We apply the following formula ${ }^{34)}$ for the inverse Laplace transformation to the last term in eqn. (39):

$L^{-1}\left[s^{\nu} \exp (-a \sqrt{s})\right]=2^{-\nu-1 / 2} \pi^{-1 / 2} \exp \left[-a^{2} /(8 t)\right]$

$$
\times t^{-\nu-1} U\left(-2 \nu-1 / 2, a(2 t)^{-1 / 2}\right)
$$

where $U$ denotes the parabolic cylinder func$t_{i o n}{ }^{35)}$ and $L^{-1}$ is the operator of the inverse Laplace transformation. Then the inverse Laplace transformation of eqn. .(39) leads to $\mathrm{I} /(\mathrm{nFc} D \mathrm{Db})=(\pi \theta)^{-1 / 2}+1$

$$
-\left(2^{3 / 4} / \pi\right) \theta^{3 / 4} \exp [-1 /(8 \theta)] U\left(2,(2 \theta)^{-1 / 2}\right)+\cdots(41)
$$

where $\theta$ is the dimensionless electrolysis time, given by

$\theta=\mathrm{Dt} / \mathrm{w}^{2}$

\section{RESULTS AND DISCUSSION}

Equation (41) expresses the dimensionless chronoamperometric curve at the microband electrode. The first term in eqn. (41) expresses the cottrellian component while the second and the succeeding terms denotes the diffusional edge effect. The first and second terms are identical with those which have been derived for evaluation of the edge effect in seminfinite diffusion at an extremely thin sheet electrode by Oldham ${ }^{25)}$. It is worth while to note that the second term is independent of time. This is also the case of the microsphere", the microdisk ${ }^{2)}$ and the microcylinder ${ }^{8)}$ electrodes. In Fig. 3 , the dimensional chronoamperometric curve calculated from eqn. (41) is shown, together with the cottrellian curve and the curve calculated

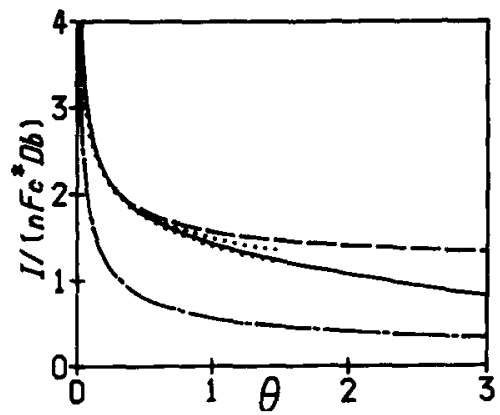

Fig. 3. Dimensionless chronoamperometric curves, (-) calculated from eqn. (41), (-...) from the cottrell equation, (--) from the first two terms of eqn. (41), and (...) from the digital simulation. 
from the first two terms in eqn. (41).

Numerical values of the parabolic cylinder function were obtained by the interpolation of values in the table $e^{36)}$ of this function.

The contribution of the edge effect can be evaluated from the ratio of eqn. (41) to the cottrell equation. When $\theta=3.2 \times 10^{-5}, 1.3 \times 10^{-4}$, 8. $0 \times 10^{-4}, 3.2 \times 10^{-3}$ and $1.3 \times 10^{-2}$, the edge effect enhances the cottrell current by $1 \%, 2 \%$, 5\% $10 \%$ and $20 \%$, respectively. These values corresponds, respectively, to $w=5.6,2.8$, 1. $1,0.56,0.29 \mathrm{~mm}$ at $t=1 \mathrm{~s}$ and $D=10^{-5} \mathrm{~cm}^{2} \mathrm{~s}^{-1}$.

We carried out the digital simulation, following the procedure proposed by Feldbero ${ }^{37)}$. We discretized the diffusion space into $50 \times 50$ $100 \times 100$ divisions. The ratio of the time increment to the area of the subspace, i.e., the dimensionless diffusion coefficient, was taken to be 0.249 . When $\theta<0.4$, values of the dimensioniess current thus computed were independent of the number of the divisions.
On the contrary, those for $\theta>0.4$ vary slightly with the number of the divisions. In Fig. 1 , shown is a concentration profile thus obtained at $\theta=0.1$. In Fig. 3 , the computed values of the dimensionless current are plotted against $\theta$. They fall in a domain interposed with two dotted curves. The curve calculated from eqn. (41) is within the interposed domain. This indicates that the analytical equation (41) is correct.

We have derived ean. (41) under the condition that values of $\theta$ are small. In order to discuss the validity of ean. (41) specifically, it is necessary to evaluate the upper limit of $\theta$. Unfortunately, unambiguous determination of the upper limit has failed at the present stage because an expansion for large values of $\theta$ has not been derived yet. In our intuitive estimation, the upper limit of $\theta$ is ca. 2.5 .

\section{REFERENCES}

1) W. R. Wightman, Anal. Chem., 53, 1125A (1981).

2) K. Aoki and J. Osteryoung, J. Electroanal. Chem., 122, 19 (1981): 160, 335 (1984).

3) K. Aoki, K. Akimoto, K. Tokuda and H. Matsuda, J. Electroanal. Chem., 171,219 (1984).

4) K. Aoki, K. Akimoto, K. Tokuda and H. Matsuda, J. Electroanal. Chem., 182, 281 (1985).

5) J. J. O'Dea, M. Wojclechowski, J. Osteryoung and K. Aok1, Anal. Chem., 57, 954 (1985).

6) D. P. Whelan, J. J. O'Dea, J. Osteryoung and K. AokI, J. Electroanal. Chem., 202, 23 (1986).

7) K. R. Wehmeyer, M. R. Deakin and R. M. Wightman, Anal. Chem., 57, 1913 (1985).

8) K. AokI, K. Honda, K. Tokuda and H. Matsuda, J. Electroanat. Chem., 186, 79 (1985).

9) K. Aok1, K. Honda, K. Tokuda and H. Matsuda, J. Electroanal. Chem., 182, 267 (1985).

10) K. Aoki, K. Honda, K. Tokuda and H. Matsuda, J. Electroanal. Chem., 195, 51 (1985).

11) S. Sujaritvanichpong, K. AOK1, K. Tokuda and H. Matsuda, J. Electroanal. Chem. 199, 271 (1986). 
12) K. Aoki, K. Tokuda and H. Matsuda, J. Electroanal. Chem. $\underline{206}, 47$ (1986).

13) M. R. Deakin, J. Electrochem. Soc., 132, 469C (1985).

14) E. Schmacher, W. Thormann and D. Arn, "Analytical Isotachophoresis", edited by F. M. Everaerts, Amsterdam. 1981, pp. 33-39.

15) W. Thormann, D. Arn and E. Schumacher, Sep. Sci. Technol., 19, 995 (1985).

16) W. Thormann and D. Arn, Electrophoresis, $\underline{5}, 323$ (1984).

17) W. Thormann, G. Twitty, A. Tsai and M. Bier, "Electrophores is ' $84^{\text {", }}$ edited by V. Neuhoff, Verlag Chemie, Weinheim, 1984, pp. 114-117.

18) W. Thormann, R. A. Mosher and M. Bier, "Electrophores is '84", edited by V. Neuhoff, Verlag Chemie, Weinheim, 1984, pp. 118-121.

19) W. Thormann, P. van den Vosch and A. M. Bond, Anal. Chem., 57, 2764 (1985).

20) H. S. White, G. P. Kittlisen, M. S. Wrighton, J. Am. Chem. Soc., 106, 5375 (1984).

21) G. P. Kittlisen, H. S. White, M. S. Wrighton, J. Am. Chem. Soc., 106, 7389 (1984).

22) E. W. Paul, A. J. Ricco, M. S. Wrighton, J. Phys. Chem., 89, 1441 (1985).

23) J. W. Thackeray, H. S. White, M. S. Wrighton, J. Phys. Chem., 89, 5133 (1985).

24) M. S. Wrighton, Comments Inorg. Chem., 4, 269 (1985).

$25)$ G. P. Kittlisen, H. S. White, M. S. Wrighton, J. Am. Chem. Soc., 107, 7373 (1985).

26) C. E. Chidsey, B. J. Feldman, C. Lundgren, R. W. Murray, Anal. Chem., 106, 5375 (1986).

27) K. R. Wehmeyer, M. R. Deakin and R. M. Wightman, Anal. Chem., 57, 1913 (1985).

28) K. B. Oldham, J. Electroanal. Chem., 122, 1 (1981).

29) B. Noble, "Methods Based on the Wiener-Hopf Technique", Pergamon Press, London, 1958.

30) K. Aoki, K. Tokuda and H. Matsuda, J. Electroanal. Chem., in press.

31) H. S. Carslaw and J. C. Jaeger, "Conduction of Heat in Solids", Oxford University Press. London, 1947, p. 336.

32) E. T. Whittaker and G. N. Watson, "A Course of Modern Analysis", Cambridge at the University Press, 1973 , p. 105.

33) F. G. Tricomi, "Integral Equations", Dover Publications, Inc, New York, 1985, p. 49.

34) G. E. Roberts and H. Kaufman, "Table of Laplace Transforms", Saunders Company, Philadelphia, 1966, p. 247.

35) M. Abramowitz and I. A. Stegun (editors), "Handbook of Mathematical Functions", Dover Publications, Inc, New York, 1970, p. 686.

36) M. Abramowitz and I. A. Stegun (editors), "Handbook of Mathematical Functions", Dover Publications, Inc, New York, 1970, p. 710.

37) S. W. Feldberg, Electroanalytical Chemistry, 3, 199 (1969). 